\title{
Data Replication Using Data Mining Techniques
}

\author{
P. Elango ${ }^{1}$, K. Kuppusamy ${ }^{2}$ and N. Prabhu ${ }^{3}$ \\ ${ }^{1}$ Research Scholar, ${ }^{2}$ Professor \& Head, ${ }^{3}$ Assistant Professor \\ ${ }^{1 \& 3}$ Department of Computer Science, Gobi Arts \& Science College, Tamil Nadu, India \\ ${ }^{2}$ Department of Computer Logistics, Alagappa University, Tamil Nadu, India \\ E-Mail: pitchaielango@gmail.com
}

\begin{abstract}
Database Replication is the successive electronic duplicating of information from a database in one PC or server to a database in another with the goal that all clients share a similar dimension of data. The outcome is a conveyed database in which clients can get to information significant to their assignments without meddling with crafted by others. Anyway information replication is an entrancing theme for both hypothesis and practice. On the hypothetical side, numerous solid outcomes requirement what should be possible as far as consistency: e.g., the difficulty of achieving agreement in offbeat frameworks the blocking idea of CAP hypothesis, and the requirement for picking an appropriate rightness foundation among the numerous conceivable. On the pragmatic side, information replication assumes a key job in a wide scope of settings like storing, back-up, high accessibility, wide territory content dissemination, expanding versatility, parallel preparing, and so forth. Finding a replication arrangement that is reasonable in whatever number such settings as could reasonably be expected remains an open test. Keywords: Component, Data Replication, Data Mining
\end{abstract}

\section{INTRODUCTION}

Information replication has been broadly used to improve the execution of information access in conventional wired/remote systems. With information replication, clients can get to the information without the help of system framework, and can lessen the traffic load. Having numerous imitations of a database improves accessibility since exchanges can keep on being executed by different copies should one go down. Moreover, bringing a smashed server reinforcement following a disappointment can be rearranged by replicating an imitation's state as opposed to revamping the slammed server's state from logs. The replication instrument figures out which document ought to be duplicated, when to make new copies and where the new reproductions ought to be put. Replication strategies can be delegated static and dynamic. Replication segments can in this way be exposed to reasonable substantial scale stacks in an assortment of situations, including issue infusion, while in the meantime giving worldwide perception and control .Replication is a practical method to expand accessibility and utilized for both execution and blame tolerant purposes subsequently presenting a steady exchange off among consistency and effectiveness. Replication is the most giving approach to voyaging sales reps and meandering detached clients and empowers portable clients with workstations to be refreshed with current database data when they associate and to transfer information to a server. Information is produced and afterward repeated.

\section{DATA MINING}

The procedure which examinations the information from various discernments and outlining it into valuable data is known as Data mining. It consolidates ordinary information examination system with convoluted calculations for giving out a lot of information. It has been extensively utilized and together with research in territories like databases, machine learning, insights and Artificial Intelligence. With reference to that, information mining has seen as an impact of enthusiasm from both scholastic world and Commercial use to improve the movement of envisioning and tolerating the model of the information. Information mining is the center piece of the Knowledge Discovery in Database (KDD) process, which is pivotal to determine a difficulty in an unequivocal area. As a rule, KDD is the entire movement of perceiving real, unique, potentially reasonable and in the end coherent models in information and develop it into valuable data. Information mining in KDD works on a specific calculation to get significant learning with the goal that the found learning can be connected in the connected zone to intensify working proficiency and furthermore to upgrade the nature of basic leadership. There are distinctive advances that are occupied with mining information as appeared in the fig. 1.

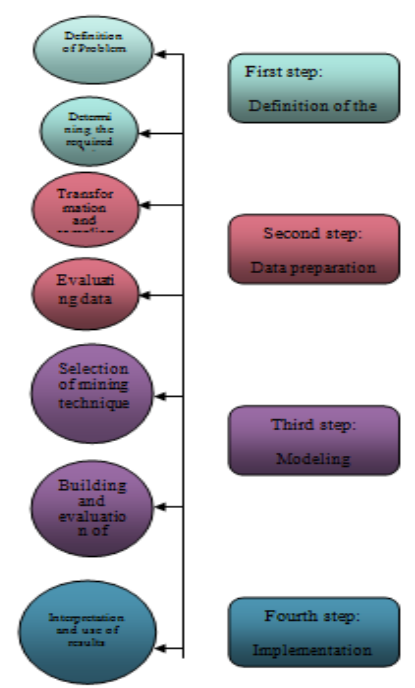

Fig. 1 Overview of Data Mining Process 


\section{OUTLINE OF CLOUD COMPUTING}

Cloud Computing represents both the software and the hardware delivered as services over the Internet. Cloud Computing is a novel idea that defines the use of computing as a efficacy, that has newly concerned major notice. There are four basic models of implementing Cloud Computing services, including:

1. Public Cloud

Platform obtainable and open to the public, regardless of whether they are individuals or organizations;

2. Private Cloud

CC infrastructure reachable to only one organization. It can be managed by the association itself or someone else who is doing that for the organization (outsourcing)

3. Community Cloud

Model of implementation that provides the ability for more organizations to share the same CC structure. Infrastructure supports special communities that have common interests, needs and security requirements

4. Hybrid Cloud

Model, which consists of two or more previously, discussed types of the establishment of CC structure which remain unique and independent entities, but with a certain kind of reciprocal link, in order to achieve mobility of data between them.

\section{FUTURE SCOPE OF CLOUD DATA MINING}

Information mining is utilized in different applications, for example, Health care, Student the executives, arithmetic, Science, in different site. The information mining in Cloud Computing enables associations to unify the administration of programming and information stockpiling, with confirmation of effective, solid and secure administrations for their clients. Here we investigate the how the information mining instruments like SAS, PAS and IaaS are utilized in distributed computing to extricate the data. Driving distributed computing suppliers Amazon Web Services, Windows Azure, Open Stack. Individuals utilize this component to manufacture data posting, get data about various points via looking in gatherings and so forth. Organizations utilizes this administration to perceive what sort of data is gliding in the internet for their items or administrations and take activities dependent on the information displayed The data recovery pragmatic model through the multi-operator framework with information mining in a distributed computing condition has been proposed. It is notwithstanding, prescribed that clients ought to guarantee that the demand made to the IaaS is inside the extent of incorporated information distribution center and is clear and straightforward. In this way, making the work for the multi-specialist framework simpler through use of the information mining calculations to recover important data from the information stockroom. Distributed computing enables the clients to recover important data from for all intents and purposes coordinated information stockroom that lessens the expenses of foundation and capacity.

\section{RESULTS AND DISCUSSION}

In our proposed philosophy, the information replication for choice supporting framework is depicted. The usage was done in JAVA. Here in our proposed part, the engineered database is utilized. Timeouts are best when a critical level of the transient disappointments can be overlooked, which is subject to the downtime dispersion. In any case, for sturdiness to stay high, the normal hub lifetime should be essentially more prominent than the timeout. To assess this situation where timeouts ought to have sway, we played out a test utilizing a manufactured follow where we changed the fix edge and the hub timeout. Since the framework would perceive hubs returning after a lasting disappointment and quickly lapse all pending timeouts for these hubs, we doled out new characters to such hubs to permit long timeouts to terminate typically. Table I demonstrates the consequences of this recreation the absolute bytes sent as an element of timeout.

TABLE I COMPARISON OF TIMEOUT ON BANDWIDTH

\begin{tabular}{|l|c|c|}
\hline Time Out (hours) & Full Replication & Partial Replication \\
\hline 0.1 & 18 & 25 \\
\hline 1 & 15 & 22 \\
\hline 10 & 12 & 19 \\
\hline 100 & 10 & 16 \\
\hline 1000 & 8 & 12 \\
\hline 10000 & 4 & 10 \\
\hline
\end{tabular}

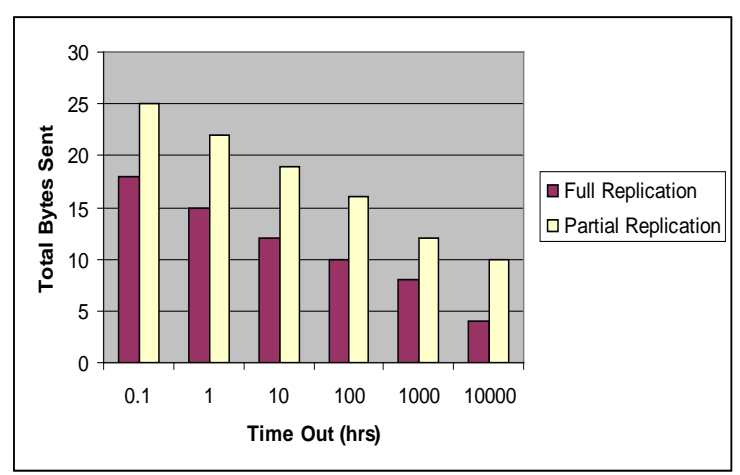

Fig. 2 Comparison of time out on bandwidth

\section{CONCLUSION}

Replication assumes a crucial job in appropriated frameworks. Frameworks disappointments regularly happen in duplicated information's because of the undesirable versatility and adaptation to non-critical failure. Choice Support frameworks (DSS) is utilized to clear the blame happens in the database by utilizing an incomplete replication with logical model. It is to comprehend the potential adaptability increases of halfway replication as for full replication. It will give better execution by utilizing a DDS-R construction. It will annihilate the framework blunders and the disseminated frameworks with database can access with numerous replication with no adaptation to internal failure. Test Results unmistakably demonstrates the 
information replication for the disseminated database utilizing choice emotionally supportive networks will give the better execution by decreasing the bogus mistakes.

\section{REFERENCES}

[1] Reza Akbarinia, Mounir Tlili, Esther Pacitti, Patrick Valduriez and Alexandre A. B. lima”, Replication in DHTs using Dynamic Groups", Lecture Notes in Computer Science, Vol. 67, No.3, pp. 1-19, 2011.

[2] Reza Akbarinia, Mounir Tlili, Esther Pacitti, Patrick Valduriez and Alexandre A. B. lima”, Continuous Time stamping for Efficient Replication Management in DHTs", Lecture Notes in Computer Science, Vol. 62, pp. 38-49, 2010.

[3] Prasanna Padmanabhan, Le Gruenwald, Anita Vallur and Mohammed Atiquzzaman”, A survey of data replication techniques for mobile ad hoc network databases, International journal on very large database”, Vol. 17, pp. 1143-1164, 2008.

[4] H. Dutta, A. Kamil, M. Pooleery, S. Sethumadhavan, and J. Demme, Distributed Storage of Large-Scale Multidimensional Electro encephalogram Data Using Hadoop and HBase”, Grid and Cloud Database Management, pp. 331-347, 2011.
[5] Hannes Muhleisen, Tilman Walther and Robert Tolksdorf”, Data Location Optimization for a Self-Organized Distributed Storage System", In Proc. of the Third World Congress on Nature and Biologically Inspired Computing (NaBIC), pp. 176 - 182, Oct. 2011.

[6] Yi Lin, Bettina Kemme, Marta Patino Martinez, and Ricardo Jimenez Peris", Consistent Data Replication: Is it feasible in WANs", Information Science and Computer communication, 2004.

[7] Zahia Guessoum, Jean-Pierre Briot, Nora Faci and Olivier Marin”, Towards reliable multi-agent systems: An adaptive replication mechanism”, Journal of Multi agent and Grid Systems, Vol.6, No. 1, pp. 1-24, 2010.

[8] Byung-Gon Chun, Frank Dabek, Andreas Haeberlen, Emil Sit, Hakim Weatherspoon, M. Frans Kaashoek, John Kubiatowicz and Robert Morris”, Efficient Replica Maintenance for Distributed Storage Systems", In Proc. of the Symposium on Networked Systems Design and Implementation, 2006

[9] Michael H. Zack, "The role of decision support systems in an indeterminate world”, Decision Support Systems, Vol. 43, No. 4, pp. 1664-1674, 2007.

[10] Sanjay Kumar Tiwari and A.K.Sharma”, Management Issues in Replicated Distributed Real Time Database”, International Journal of Advance in Science and Technology, Vol. 3, No. 1, pp. 75-89, 2011. 